\title{
Public Health Genomics and Genetic Test Evaluation: The Challenge of Conducting Behavioural Research on the Utility of Lifestyle-Genetic Tests
}

\author{
Saskia C. Sanderson ${ }^{a-c}$ Jane Wardle ${ }^{b}$ Steve E. Humphries ${ }^{c}$ \\ ${ }^{a}$ Social and Behavioral Research Branch, National Human Genome Research Institute (NHGRI), National Institutes

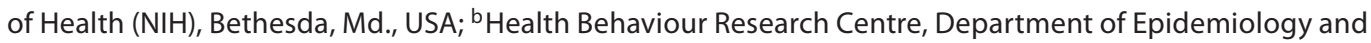 \\ Public Health, and ${ }^{\mathrm{C} C e n t r e ~ f o r ~ C a r d i o v a s c u l a r ~ G e n e t i c s, ~ D e p a r t m e n t ~ o f ~ M e d i c i n e, ~ U n i v e r s i t y ~ C o l l e g e ~ L o n d o n, ~ U K ~}$
}

\author{
Key Words \\ Genetic testing $\cdot$ Lifestyle $\cdot$ Health behaviours $\cdot$ Clinical \\ utility $\cdot$ Public health genomics
}

\begin{abstract}
Human genetics research is increasingly concerned with multifactorial conditions such as diabetes and heart disease, which are influenced not only by genetic but also lifestyle factors such as diet and smoking. Although the results of 'lifestyle-genetic' tests using this information could conceivably motivate lifestyle changes in the future, companies are already selling such tests and related lifestyle advice commercially. Some academics and lobby groups have condemned the companies for selling these tests in advance of scientific support. Others are concerned that the tests may not motivate lifestyle improvements, instead causing distress in people receiving adverse test results and complacency in those receiving reassuring results. There is currently no regulatory oversight of genetic test utility, despite consensus in the Public Health Genomics community that clinical utility (including psychological and behavioural impact) of all emerging genetic tests should be evaluated before being introduced for individual use. Clearly, empirical data in this area is much needed, to inform understanding of the potential utility of these tests, and of whether stricter
\end{abstract}

\section{KARGER \\ Fax +41613061234 \\ E-Mail karger@karger.ch}

(C) 2008 S. Karger AG, Basel

www.karger.com regulation of commercial exploitation is needed. In this article, we review the current situation regarding lifestyle-genetic tests, and discuss the challenges inherent in conducting this kind of behavioural research in the genomics era.

Copyright $\odot 2008$ S. Karger AG, Basel

\section{Introduction}

The sequencing of the human genome has led to an explosion of genetic association studies in recent years [1-3]. Genetic association studies aim to detect association between one or more genetic polymorphisms and a quantitative characteristic or disease $[4,5]$. The genetic variations studied include not only rare mutations that have a major impact on disease risk, but also common gene variants, found at high frequencies in the population, which have a much more modest impact on disease risk. This has transformed human genetics research by shifting the focus from rare, single-gene disorders, to common, complex conditions such as heart disease, diabetes, obesity, and addiction [6].

These developments raise the question 'What do you do with a gene when you find one?' [7]. There are four potential applications of genetic information in the treatment and prevention of complex diseases [6]. First, it in-
Prof. Steve Humphries

Centre for Cardiovascular Disease, Rayne Building

5 University Street

London WC1E 6JJ (UK)

Tel. +44 207679 6962, Fax +44 207679 212, E-Mail rmhaseh@ucl.ac.uk 
creases understanding of the aetiology and causal pathways to the disease. Second, it may help develop new drug treatments and identify new pharmacological targets. Third, it will help stratify patient groups so that individuals are only given a drug treatment if it is known to be effective and safe for their personal genotype (i.e. pharmacogenomics) [6]. Fourth, providing individuals with DNA-based information about their susceptibility to developing a complex condition, before it develops, may motivate them to make lifestyle changes to reduce their future risk of developing the condition [6-10].

Although most research has focused on the first three translational areas, namely on the idea of developing drug treatments and pharmacogenomics, there are important questions about using genetic information to motivate lifestyle changes. For example, in the case of the genetics of diabetes [6,11-13], such huge proportions of the population are becoming obese and therefore diabetic, it is likely to be too much of an economic burden to place all these people on medication. Furthermore, given the importance of environmental effects in the current obesity and diabetes epidemics, it would be preferable to motivate at least some of the at-risk individuals to change their behaviour before they become obese, and genetic information may be helpful in identifying these people and then enhancing their motivation to change.

A shift of focus towards complex conditions could have a significant impact on how genetics research is expected to improve health in the future. Because complex conditions such as diabetes and heart disease are influenced by both genetic and environmental factors, there is increasing interest in the potential for using genetic test information about an individual's disease susceptibility to aid clinical management, not only by developing better pharmacological interventions, but by improving risk-reducing behavioural interventions, e.g. improving dietary habits, physical activity and smoking cessation $[8,9,10]$. But there is also controversy. For example, Janssens, Khoury and colleagues recently drew attention to how, when the Iceland-based Decode company published their report on the association of the TCF7L2 genotype with type 2 diabetes[14], the company CEO Kari Stefansson told the New York Times that the discovery could lead to a diagnostic test to identify people who carry the variant gene, and also that people who knew of their extra risk would be motivated to avoid the lifestyle habits that lead to diabetes $[15,16]$ : 'An immediate practical consequence of the discovery, said Decode's chief executive, Kari Stefansson, would be to develop a diagnostic test to identify people who carry the variant gene. If people knew of their extra risk, they would have an incentive to stay thin and exercise, he said (New York Times, January 16, 2006).' Janssens and others [8] have asked the reasonable question, Where is the evidence to support the claim that people will use genetic information as a motivator to change their behaviour?

Research is only just beginning to emerge which will provide evidence on this issue, but there is also considerable scepticism about whether genetic risk information will really motivate and sustain lifestyle changes, or whether it will instead 'medicalize' multifactorial diseases even more than they are already, and distract attention away from modifiable lifestyle risk factors [17-20]. Khoury and colleagues [7,21-24] have written widely on this topic, and one of their conclusions is that there is a paucity of data available regarding whether genetic risk can be a motivator for, or a hindrance to, behavioural change [21, p. 452]. Along with their colleagues comprising the Genome-Based Research and Population Health International Network (GRAPH Int) and others, they have proposed that new and emerging genetic tests need to be assessed systematically, and this should include the impact on psychological wellbeing and the potential to improve health via lifestyle change interventions [21, 25].

In this article, we discuss the issues raised by the new 'lifestyle-genetic tests' which for the purposes of this article we define as 'DNA-based tests for common gene variants reported to be associated with susceptibility to complex, lifestyle-related conditions, the results of which are fed back to individuals with the goal of increasing motivation to make lifestyle improvements'. We discuss the commercial availability of lifestyle-genetic tests, review the limited evidence on whether such tests might motivate or demotivate lifestyle change and discuss the challenges for behavioural researchers working in this field.

\section{The Commercial Availability of Lifestyle-Genetic Tests}

Lifestyle-genetic tests are already being sold direct-tothe-public by commercial companies, who claim to test individuals for genetic susceptibility to complex conditions such as heart disease, and offer lifestyle advice on the basis of the test results (e.g. www.genovations.com; www.sciona.com: accessed 22.2.08). Many of the tests are based on nutrigenomics, i.e. the study of the relationship between nutrient intake and how genes function $[26,27]$. 
The companies claim that the gene-disease associations are robust and that their consumers use the information positively to optimize their lifestyle as part of their desire to improve their health [28]. However, they have been criticized by scientists $[23,29,30]$ and lobby groups (for a GeneWatch UK briefing on this topic see: http://www. genewatch.org/HumanGen/Sciona/ScionaBrief.rtf), because the tested gene variants are only having at best a modest impact on the risk of the specified condition, and often the effect has not been adequately replicated. Major concerns have also been raised about the psychological impact of the tests [23], and questions have been raised about the evidence to support the claim that the tests lead to improvements in health behaviours [31].

\section{Current Regulation of Lifestyle-Genetic Tests in the UK}

There has been debate about whether genetic tests for complex disease susceptibility are adequately regulated at the present time [32], and a call for stricter government regulation in the UK from GeneWatch UK. The most relevant UK government bodies are the Department of Health's Medicines and Healthcare products Regulatory Agency (MHRA: www.mhra.gov.uk, accessed 22.2.08) and the Human Genetics Commission (HGC: www.hgc. gov.uk, accessed 22.2.08). The MHRA was set up in 2003 to safeguard public health by ensuring that medicines and medical devices for human use, sold or supplied in the UK, are of an acceptable standard of safety, quality, efficacy, and performance. This includes the use of human genetic material for medical purposes. Regulation of the validity of genetic tests, i.e. the laboratory part of the process, is now in place. However, assessing utility (i.e. safety and improvement in health for individuals taking the lifestyle-genetic tests) does not appear to fall within its remit and the MHRA has taken no action regarding evaluating utility. The HGC is the government's advisory body on human genetics, but has no statutory powers. There is, therefore, little regulation of lifestyle-genetic testing in terms of whether the tests are likely to be beneficial or harmful for the recipients of the results.

Whether stricter regulation regarding utility is needed should clearly depend on evaluation of the balance of risks and benefits to individuals taking the tests. As alluded to above, risks and benefits can be seen as part of the concept of 'clinical utility'. Although there has recently been debate about the correct definition [22], clinical utility here can be seen as the extent to which the test is safe and leads to health benefits to the patient [33], e.g. the extent to which testing increases behaviour change without causing psychological harm. As Khoury et al. [34, p. 200] state: 'Ultimately, the utility of genetic information will depend on whether a genetic test can lead to a medical, behavioral, or environmental intervention that prevents disease and disability.'

Investigating the clinical utility of lifestyle-genetic testing for common gene variants of modest risk impact will make it possible to determine whether such tests could be argued to produce public benefit. If giving genetic test results can be shown to motivate behaviour change without causing psychological adverse effects, then utility may be high [35]. But if research showed there were adverse effects on behaviour and psychological wellbeing, this would have important policy implications. There is therefore an urgent need for research into the psychological and behavioural impact of commerciallyavailable lifestyle-genetic tests.

\section{Will Lifestyle-Genetic Tests Motivate People to Change Their Behaviour?}

At present, only a handful of studies have examined the impact of genetic testing for susceptibility to lifestylerelated conditions (obesity, heart disease, and lung cancer) on motivation to make related lifestyle improvements (weight loss and smoking cessation). These are reviewed below.

\section{Genetic Testing and Weight Loss}

One study to date has examined the impact of DNA testing individuals for a gene associated with weight gain and energy expenditure (the b3-adrenergic receptor (b3AR) gene) [36]. The 30 obese women in this study showed increased confidence in their ability to lose weight, regardless of whether their personal genetic test result indicated that they were a carrier or non-carrier of the adverse b3AR gene variant (although interpretation of this study is limited by its lack of control group). One experimental study has looked at the impact of obesityrelated genetic testing including randomization to a nongenetic test control group, but using hypothetical rather than real genetic test results [37]. In this study of 249 healthy, non-obese individuals, those who received high genetic risk test results for obesity were more motivated to eat a healthy diet than those who received low genetic risk results, but genetic risk feedback was no more or less motivating than non-genetic risk feedback. 


\section{Genetic Testing and Smoking Cessation}

Three studies have examined the impact of DNA testing for lung cancer susceptibility on motivation to quit smoking. Smokers undergoing DNA testing for lung cancer susceptibility who received results indicating increased risk showed greater perceived risk of lung cancer, and greater belief that quitting will reduce their risk of developing lung cancer $[38,39]$, than smokers receiving non-genetic or low-risk results. Smokers undergoing DNA testing for lung cancer susceptibility have also reported greater smoking cessation in the short term [40], or in the presence of telephone counselling [41] than smokers not undergoing DNA testing.

Finally, one study used hypothetical scenarios with 261 smokers to examine the impact of DNA testing for heart disease susceptibility on motivation to quit smoking, and found that smokers were more motivated to quit smoking, and had greater belief that quitting smoking would reduce their disease risk [42], than those receiving results based on non-DNA-based (oxidative stress) testing.

Although the results of these studies present a somewhat mixed picture, overall they suggest that genetic testing for complex conditions such as heart disease could motivate behaviour change or influence important predictors of behaviour change such as confidence that behaviour change will reduce disease risk, without causing adverse effects such as fatalism and false reassurance [16]. However, these are only the first steps in establishing the full behavioural impact of, and identifying groups who might benefit more or less from, genetic test information.

\section{Challenges Facing Researchers Evaluating Lifestyle-Genetic Tests and Some Solutions}

The challenges faced by behavioural researchers attempting to conduct responsible research in this field are: (1) having sufficient knowledge about genetics to be able to make judgements about whether specific common gene variants are appropriate to feed back to individuals; (2) having sufficient knowledge about genetics to be able to communicate with individuals about the genes included in the tests; (3) being able to conduct research with real genetic tests without being judged to be using them prematurely and irresponsibly in the same way as the commercial lifestyle-genetic testing companies, and (4) conducting large enough studies to get adequate statistical power to detect subtle changes in behaviour. This last point is important because most existing interventions to modify behaviour almost always result in only small improvements in behaviour. We propose two key recommendations which would help meet these challenges.

\section{A New Generation of Transdisciplinary Researchers Is Needed}

First, to take the field forward, it will be necessary to train a new generation of health behaviour researchers who are able to understand complex genetic information in order that they might competently communicate the information to patients and participants, information which is, by definition, extremely complex. As Wang et al. [43, p. 695] state, '[p]ractitioners in health education and behaviour are uniquely positioned to contribute to public health genetics by developing relevant intervention strategies'. These researchers must be trained not only in health psychology and behavioural research methods, but will also require in-depth training at a graduate and postgraduate level in genetics. This will enable them to make independent judgements about which specific gene variants to include in their research tests, as well as sufficiently competent to communicate with research participants about genetics and the meaning of their DNA-based test results.

This is one of the key aims of the new field of Public Health Genomics (or Public Health Genetics), which was established to promote the goal of achieving the effective translation of genome-based knowledge for the benefit of population health [21]. A number of Masters and $\mathrm{PhD}$ courses have been established in the USA to train a new generation of researchers in public health genomics, who are potentially able to work equally well in genetics and social sciences, such as those at the University of Michigan (http://www.sph.umich.edu/genomics/) and the University of Washington (http://depts.washington. edu/phgen/).

Formal public health genomics teaching or research programmes have yet to be implemented in the UK. The only UK Public Health Genetics Unit (www.phgu.org.uk) does not have a formal postgraduate teaching programme. We believe that the UK would benefit from the introduction of Public Health Genomics Masters and $\mathrm{PhD}$ programmes of research and training similar to those recently introduced in the USA. One of the results of this would be a new cadre of researchers who would be fully equipped to conduct research into the utility of emerging human genetic technologies, including lifestyle-genetic tests. 
Stepwise Research Frameworks Should Be

Implemented and Encouraged

Second, researchers conducting behavioural research on lifestyle-genetic tests should be aware that they may be criticized for conducting empirical research prematurely. However, there are many different methodologies available that may circumvent these criticisms. The gold standard for evaluating the impact of new pharmacological interventions designed to improve health is the RCT. An important feature of RCTs is that they should be large enough to have sufficient statistical power to detect differences in definitive and measurable clinical endpoints. There is increasing consensus that interventions designed to improve health by changing behaviours such as smoking, exercise and diet, should be subject to the same rigorous evaluation as pharmacological interventions [44]. Lifestyle-genetic tests would appear to merit the same rigorous evaluation $[23,45]$.

The difficulty is that conducting large RCTs with hundreds if not thousands of participants undergoing lifestyle-genetic testing may be irresponsible, because such studies may necessitate that the genetic testing process be done from a distance (e.g. via the post) and thus not in face-to-face settings, and because the validity and reliability of the gene variants included in lifestyle-genetic tests have yet to be demonstrated. Although one study has shown that postal lifestyle-genetic test results have no more adverse effects on individuals than face-to-face results [46], we believe it is best to be cautious before embarking on large studies of this kind, not least because of major uncertainties about people's understanding of genetic risk information [47].

To avoid accusations that large-scale RCTs have been conducted prematurely or inappropriately, we propose that researchers examining the clinical utility of emerging lifestyle-genetic tests go through a rigorous structured, phased protocol, of the kind suggested in the UK Medical Research Council's (MRC) stepwise framework for development and evaluation of RCTs for complex interventions to improve health (http://www.mrc.ac.uk/ pdf-mrc_cpr.pdf; accessed 3.10.06) [44]. Here we briefly summarize the four key phases of the MRC framework, and discuss each in the context of lifestyle-genetic tests.

(1) Preclinical or Theoretical Phase. This phase comprises a review of the theoretical basis for the intervention and of previous empirical evidence. Although this is a prerequisite of all research, the debate around the potential impact of genetic testing for behaviour-related diseases has been, until recently [8], relatively devoid of a theoretical framework. The current debates about genet- ic testing and whether people will respond to test results by being, for example, falsely reassured about their health risks, would be greatly enhanced by increased consideration and enhancement of existing models of health behaviour, such as the common-sense model of self-regulation, and protection motivation theory $[8,48]$.

(2) Phase I: Defining Components of the Intervention. Phase I comprises qualitative testing through focus groups or case studies, or quantitative preliminary surveys, to identify the components of the intervention, and the underlying mechanisms by which they will influence outcomes.

Lifestyle-genetic tests are complex interventions because there is a huge amount of variability possible in how the testing process is delivered. Who should give the test results? How should they be communicated? Verbally or visually? In relative or absolute risks? How do people understand and perceive genetic risks? How can the test results be communicated to enhance understanding? What lifestyle or other risk-reducing advice should accompany the test results? These are just a few of the many questions that need to be considered when developing interventions to improve health which incorporate genetic testing.

A handful of Phase I studies have started addressing these questions regarding lifestyle-genetic tests. For example, one study used focus groups to explore people's understanding of a 'gene for heart disease' [49], and found that 'genetic determinism' was not a major feature of public perceptions of heart disease genetics, despite earlier concerns [50]. Although very preliminary, these results might suggest that concerns about genetic determinism or fatalism should not necessarily be viewed as a barrier to introducing genetic testing for common conditions in the public domain. In addition, two studies used survey methods to address whether people in the general public, away from the clinic setting, were interested in taking genetic tests for complex diseases linked to lifestyle (heart disease and (lung) cancer), and found that public interest in taking genetic tests was high, and that individual characteristics such as family history of the disease predicted who was interested in testing $[51,52]$. In addition, a few experimental studies have used hypothetical scenarios to demonstrate how manipulation of variables can influence behavioural outcomes [37, 42].

More research is clearly needed, but these types of experimental studies will ultimately help develop understanding of whether and by what mechanisms genetic testing might motivate lifestyle change and conversely the conditions under which it might have adverse effects. Phase I studies are important to conduct in the early stag- 
es of research, but are limited by their hypothetical nature.

(3) Phase II: Defining Trial and Intervention Design. In Phase II the information gathered in Phase I is used to develop the optimum intervention and study design, and is the first phase in which the actual intervention is trialled. This is an exploratory phase, one of the main aims of which is to assess the feasibility of the intervention and acceptability to users. Different versions of the intervention may need to be tested, and randomized controlled methods should be used, but the studies should be small and exploratory.

In the case of lifestyle-genetic tests, this means developing responsible protocols and conducting research studies in which individuals are randomly assigned to receive real genetic test results for common gene variants associated with complex diseases, for example, heart disease, lung cancer, or diabetes, but on a small scale. This should ensure that care is taken to ensure the wellbeing of the study participants, and it allows rigorous trialling of various ways of delivering the intervention. Only two Phase II studies have been conducted which have used real genetic tests for common gene variants, one with 61 participants and the lung cancer-associated glutathione S-transferase M1 (GSTM1) gene [40], and one with 30 participants and the obesity-associated b3AR gene (although this study did not have a control group) [36]. The results of both studies suggested that the individuals in these trials were not distressed or misled by their test results, and provided preliminary evidence suggesting that lifestyle-genetic testing may indeed enhance motivation to improve lifestyle.

We suggest that whilst uncertainties remain about the validity and reliability of the genetic variants being tested for, Phase II studies should be encouraged, as long as researchers have sufficient evidence from meta-analyses of association studies to support the use of the specific gene variants being used in the tests, have sufficient data from Phase I to inform the design of the study, and honestly communicate the uncertainties around the genetic information to participants. We propose that small RCTs are valid despite potentially being underpowered to detect some differences in actual behaviour, because they allow researchers to use real genetic tests in controlled research settings, and to move on from Phase I hypothetical studies which whilst useful do not use real DNA tests, without embarking prematurely on inappropriate and costly large Phase III RCTs. Large Phase III trials should only be embarked upon once there is greater confidence in the associations between the gene variants and the diseases be- ing tested for, and once enough data has been published from Phases I and II to demonstrate the safety of a largescale trial.

(4) Phase III: The Main Randomized Controlled Trial. The design of a major trial of lifestyle-genetic tests needs to address all the issues of an RCT, such as sufficient sample size to detect the expected modest effects, inclusion and exclusion criteria, methods of randomization, and accurate measures of the behavioural and health-related end points.

Only two Phase III trials have been conducted in the field of lifestyle-genetic tests. Both examined the impact of genetic tests for lung cancer susceptibility on smoking cessation $[39,41]$. The results of these studies have provided a mixed picture, one suggesting genetic testing is motivational [41], and one not [39]. More Phase III data will ultimately be needed on lifestyle-genetic testing, but these kinds of studies should only be conducted after rigorous trialling in Phases I and II. This should increase confidence in the appropriateness of these studies being conducted, and the willingness of funding bodies to provide financial support.

\section{Summary, Implications and Conclusions}

Research evaluating the behavioural and psychological impact of lifestyle-genetic tests is needed because commercial companies are already selling lifestyle-genetic tests direct-to-the-public; it is not known whether they might be helpful in improving the health behaviours of the public or cause harm by reducing motivation to make such improvements, and there is not enough data available on utility at present to inform policy regarding the regulation of these tests.

Although the principles behind direct-to-consumer lifestyle-genetic tests might be sound, the tests are yet to meet the clinical utility standards necessary (in addition to the other two core evaluation criteria, analytic validity and clinical validity [53]) to justify the clinical, community or commercial use of lifestyle-genetic tests. No commercially available lifestyle-genetic tests have yet been shown to have clinical utility, that is, to be both safe for the individual taking the test and to have the long-term consequence of reducing that individual's subsequent disease risk by altering lifestyle behaviours.

However, the strict regulation called for by some lobby groups may be both an overreaction and unrealistic, and is arguably an example of undesirable 'genetic exceptionalism'. We do not legislate against purveyors of (pre- 
sumed) non-harmful products, such as aromatherapy oils, even if benefit is unproven or absent (the so-called 'snake-oil principal') [54]. In a world where millions of people buy dubious medical devices or products every day, and where regulation is increasingly difficult to enforce because of the worldwide internet, a more flexible approach is in most cases deemed permissible if there is no documented harm attributed to the product. This seems increasingly possible for the types of lifestyle-genetic tests currently being offered and that are on the horizon, but clearly what is needed is more research of the kind reviewed in this article.

One additional implication of direct-to-consumer marketing of health-related genetic technologies such as lifestyle-genetic tests is that increased public as well as professional education about genetics and gene-environment interactions is needed [55]. At present, the public views genetic susceptibility as playing only a limited role in common human diseases which are induced by environmental and behavioural factors, but in which genetic factors also play a significant role [56]. As stated by Morris et al. [56], increasing awareness of the role of genetic factors in complex diseases will be necessary for translating gene discovery into effective personal and public health actions. We live in an ever-more information-led society, and rather than attempting to restrict access to tests, it might be preferable and more cost-effective, to provide the information that people need to make the appropriate choices for themselves. This could be through enforcing package labelling, as well as via websites and through newspapers and magazines. There is a strong case for insisting that the commercial companies disclose which gene variants they are testing for, much in the way that food manufacturers have to label the ingredients their products contain. At present, some companies do provide easily accessible, publicly available information on the gene variants they test for (e.g. www.ilgenetics. com: accessed 23.5.08) but others do not (e.g. www.sciona.com: accessed 23.5.08). We agree with Haga et al. [23, p. 349] that companies should be required to provide upto-date figures on the strength of association between the genes being tested for and the disease or condition of interest, both on the products and on the company websites. This information would provide health professionals and consumers with complete information about what a test can and cannot provide so that they may make an informed decision [23].

In conclusion, to provide much needed policy guidance on the clinical utility of lifestyle-genetic tests, ethically sound empirical research must be conducted which examines the behavioural impact of such testing, but which does not lead to the researchers undertaking the research being accused of offering genetic tests to individuals prematurely. Improving the genetic literacy of the health behaviour researchers conducting the research will make a significant contribution towards this endeavour. In addition, the MRC framework for the development and evaluation of complex interventions [44] provides a pragmatic framework and cautious, stepwise protocol, within which large RCTs are only conducted following a series of preliminary interviews, surveys, and smaller exploratory RCTs. Phased behavioural research will provide data essential to the assessment of the clinical utility of lifestyle-genetic tests, and should inform policy initiatives concerning the provision of lifestyle-genetic tests for susceptibility to complex diseases.

\section{References}

1 The International HapMap Consortium: A haplotype map of the human genome. $\mathrm{Na}$ ture 2005;437:1299-1320.

-2 Sachidanandam R, et al: A map of human genome sequence variation containing 1.42 million single nucleotide polymorphisms. Nature 2001;409:928-933.

3 Collins FS, Morgan M, Patrinos A: The Human Genome Project: lessons from largescale biology. Science 2003;300:286-290.

4 Cordell HJ, Clayton DG: Genetic association studies. Lancet 2005;366:1121-1131.

5 Davey SG, et al: Genetic epidemiology and public health: hope, hype, and future prospects: Lancet 2005;366:1484-1498.
6 Collins FS, Green ED, Guttmacher AE, Guyer MS: A vision for the future of genomics research. Nature 2003;422:835-847.

7 Khoury MJ, Davis R, Gwinn M, Lindegren $\mathrm{ML}$, Yoon P: Do we need genomic research for the prevention of common diseases with environmental causes? Am J Epidemiol 2005; 161:799-805.

8 Marteau TM, Weinman J: Self-regulation and the behavioural response to DNA risk information: a theoretical analysis and framework for future research. Soc Sci Med 2006;62:1360-1368.

9 Collins FS: The human genome project and the future of medicine. Ann NY Acad Sci 1999;882:42-55
10 Collins FS: Shattuck Lecture - medical and societal consequences of the Human Genome Project. N Engl J Med 1999;341:2837.

1 Gerich JE: The genetic basis of type 2 diabetes mellitus: impaired insulin secretion versus impaired insulin sensitivity. Endocrinol Rev 1998;19:491-503.

12 Hitman GA, Sudagani J: Searching for genes in diabetes and the metabolic syndrome. Int J Clin Pract Suppl 2004;143:3-8.

13 Lyssenko V, et al: Predictors of and longitudinal changes in insulin sensitivity and secretion preceding onset of type 2 diabetes. Diabetes 2005;54:166-174. 
14 Grant SF, et al: Variant of transcription factor 7-like 2 TCF7L2 gene confers risk of type 2 diabetes. Nat Genet 2006;38:320-323.

15 Wade N: Gene Increases Diabetes Risk, Scientists Find. New York, New York Times, 2006.

16 Janssens AC, Gwinn M, Valdez R, Narayan KM, Khoury MJ: Predictive genetic testing for type 2 diabetes. BMJ 2006;333:509-510.

17 Cooper RS, Psaty BM: Genomics and medicine: distraction, incremental progress, or the dawn of a new age? Ann Intern Med 2003; 138:576-580.

-18 Merikangas KR, Risch N: Genomic priorities and public health. Science 2003;302:599601.

19 Merikangas KR, Risch N: Will the genomics revolution revolutionize psychiatry? Am J Psychiatry 2003;160:625-635.

20 Willett WC: Balancing life-style and genomics research for disease prevention. Science 2002;296:695-698.

21 Burke W, Khoury MJ, Stewart A, Zimmern RL: The path from genome-based research to population health: development of an international public health genomics network. Genet Med 2006;8:451-458.

22 Grosse SD, Khoury MJ: What is the clinical utility of genetic testing? Genet Med 2006;8: 448-450.

23 Haga SB, Khoury MJ, Burke W: Genomic profiling to promote a healthy lifestyle: not ready for prime time. Nat Genet 2003;34: 347-350.

24 Khoury MJ, Jones K, Grosse SD: Quantifying the health benefits of genetic tests: the importance of a population perspective. Genet Med 2006;8:191-195.

25 Scheuner MT, Rotter JI: Quantifying the health benefits of genetic tests: a clinical perspective. Genet Med 2006;8:141-142.

-26 Mutch DM, Wahli W, Williamson G: Nutrigenomics and nutrigenetics: the emerging faces of nutrition. FASEB J 2005; 19:16021616.

27 Ordovas JM: Nutrigenetics, plasma lipids, and cardiovascular risk. J Am Diet Assoc 2006;106:1074-1081.

28 Roberts G, Grimaldi K: Sciona and genetic testing. Nat Genet 2003;33:121.

29 Vineis P, Christiani DC: Genetic testing for sale. Epidemiology 2004;15:3-5

30 Mykitiuk R: Caveat emptor: direct-to-consumer supply and advertising of genetic testing. Clin Invest Med 2004;27:23-32.
31 Marteau TM, Lerman C: Genetic risk and behavioural change. BMJ 2001;322:10561059.

32 Holtzman NA: Are genetic tests adequately regulated? Science 1999;286:409.

33 Holtzman NA: Promoting safe and effective genetic tests in the United States: work of the task force on genetic testing. Clin Chem 1999;45:732-738.

34 Khoury MJ, et al: Challenges in communicating genetics: a public health approach. Genet Med 2000;2:198-202.

35 Burke W, Pinsky LE, Press NA: Categorizing genetic tests to identify their ethical, legal, and social implications. Am J Med Genet 2001;106:233-240.

36 Harvey-Berino J, et al: Does genetic testing for obesity influence confidence in the ability to lose weight? A pilot investigation. J Am Diet Assoc 2001;101:1351-1353.

37 Frosch DL, Mello P, Lerman C: Behavioral consequences of testing for obesity risk. Cancer Epidemiol Biomarkers Prev 2005;14: 1485-1489.

38 Audrain J, et al: Genetic susceptibility testing in smoking-cessation treatment: oneyear outcomes of a randomized trial. Addict Behav 1997;22, 741-751.

39 Lerman C, et al: Incorporating biomarkers of exposure and genetic susceptibility into smoking cessation treatment: effects on smoking-related cognitions, emotions, and behavior change. Health Psychol 1997;16: 87-99.

40 Sanderson SC, Humphries SE, Hubbart C, Hughes E, Jarvis MJ, Wardle J: Psychological and behavioural impact of genetic testing smokers for lung cancer risk: a phase II exploratory trial. J Health Psychol 2008;13:481494.

41 McBride CM, et al: Incorporating genetic susceptibility feedback into a smoking cessation program for African-American smokers with low income. Cancer Epidemiol Biomarkers Prev 2002;11:521-528.

42 Sanderson SC, Michie S: Genetic testing for heart disease susceptibility: potential impact on intention to quit smoking. Clin Genet 2007;71:501-510.

43 Wang C, Bowen DJ, Kardia SL: Research and practice opportunities at the intersection of health education, health behavior, and genomics. Health Educ Behav 2005;32:686701.

44 Campbell M, et al: Framework for design and evaluation of complex interventions to improve health. BMJ 2000;321:694-696.
45 Gollust SE, Hull SC, Wilfond BS: Limitations of direct-to-consumer advertising for clinical genetic testing. JAMA 2002;288;17621767.

46 McBride CM, et al: Maximizing the motivational impact of feedback of lung cancer susceptibility on smokers' desire to quit. J Health Commun 2000;5:229-241.

47 Lipkus IM, McBride CM, Pollak KI, et al: Interpretation of genetic risk feedback among African-American smokers with low socioeconomic status. Health Psychol 2004;23: $178-188$.

48 Gooding HC, Organista K, Burack J, Biesecker BB: Genetic susceptibility testing from a stress and coping perspective. Soc Sci Med 2006;62:1880-1890.

49 Bates BR, Templeton A, Achter PJ, et al: What does 'a gene for heart disease' mean? A focus group study of public understandings of genetic risk factors. Am J Med Genet A 2003 119:156-161.

50 Senior V, Marteau TM, Peters TJ: Will genetic testing for predisposition for disease result in fatalism? A qualitative study of parents responses to neonatal screening for familial hypercholesterolaemia. Soc Sci Med 1999;48:1857-1860.

51 McBride CM, Lipkus IM, Jolly D, Lyna P: Interest in testing for genetic susceptibility to lung cancer among Black college students 'at risk' of becoming cigarette smokers. Cancer Epidemiol Biomarkers Prev 2005;14:29782981.

52 Sanderson SC, Wardle J, Jarvis MJ, Humphries SE: Public interest in genetic testing for susceptibility to heart disease and cancer: a population-based survey in the UK. Prev Med 2004;39:458-464.

53 Sanderson S, et al: How can the evaluation of genetic tests be enhanced? Lessons learned from the ACCE framework and evaluating genetic tests in the United Kingdom. Genet Med 2005;7:495-500.

54 Bowen DJ, Battuello KM, Raats M: Marketing genetic tests: empowerment or snake oil? Health Educ Behav 2005;32:676-685.

55 Zimmern R, Emery J, Richards T: Putting genetics in perspective requires better understanding and more rational debate. BMJ 2001;322:1005-1006.

56 Morris J, Gwinn M, Clyne M, Khoury MJ: Public knowledge regarding the role of genetic susceptibility to environmentally induced health conditions. Community Genet $2003 ; 6: 22-28$. 